\title{
Dynamics of complex-valued fractional-order neural networks
}

\author{
Eva Kaslik ${ }^{\mathrm{a}, \mathrm{b}, *}$, Ileana Rodica Rădulescu ${ }^{\mathrm{a}, \mathrm{c}}$ \\ ${ }^{a}$ Institute e-Austria Timisoara, Bd. V. Parvan nr. 4, room 045B, 300223, Timisoara, \\ Romania \\ ${ }^{b}$ Department of Mathematics and Computer Science, West University of Timisoara, \\ Romania \\ ${ }^{c}$ Faculty of Applied Sciences, University Politehnica of Bucharest, Romania
}

\begin{abstract}
The dynamics of complex-valued fractional-order neuronal networks are investigated, focusing on stability, instability and Hopf bifurcations. Sufficient conditions for the asymptotic stability and instability of a steady state of the network are derived, based on the complex system parameters and the fractional order of the system, considering simplified neuronal connectivity structures (hub and ring). In some specific cases, it is possible to identify the critical values of the fractional order for which Hopf bifurcations may occur. Numerical simulations are presented to illustrate the theoretical findings and to investigate the stability of the limit cycles which appear due to Hopf bifurcations.
\end{abstract}

Keywords: neural networks, fractional order, fractance, stability, multistability, instability, Hopf bifurcation, ring, hub

\section{Introduction}

In the last few decades, generalizations of dynamical systems using fractional derivatives instead of classical integer-order derivatives have proved to be more accurate in the mathematical modeling of real world phenomena arising from several interdisciplinary areas. It is now well-understood that fractional derivatives provide a good tool for the description of memory

\footnotetext{
*Corresponding author

Email addresses: ekaslik@gmail.com (Eva Kaslik), nicola_rodica@yahoo.com (Ileana Rodica Rădulescu)
} 
and hereditary properties of various processes, fractional-order systems being characterized by infinite memory. Phenomenological description of viscoelastic liquids (Heymans and Bauwens, 1994), colored noise (Cottone et al., 2010), diffusion and wave propagation (Henry and Wearne, 2002; Metzler and Klafter, 2000), boundary layer effects in ducts (Sugimoto, 1991), fractional kinetics (Mainardi, 1996), electromagnetic waves (Engheia, 1997), electrode-electrolyte polarization (Ichise et al., 1971), represent just a few of the many application areas of fractional derivatives.

It is important to emphasize that many qualitative properties of integerorder dynamical systems cannot be extended by simple generalizations to fractional-order dynamical systems, and hence, the analysis of fractionalorder dynamical systems is a very important field of research. For example, it has been shown (Kaslik and Sivasundaram, 2012a) that the fractionalorder derivative (of Caputo, Grunwald-Letnikov or Riemann-Liouville type) of a non-constant periodic function cannot be a periodic function of the same period, while the integer-order derivative of a periodic function is indeed a periodic function of the same period. As a consequence, exact periodic solutions do not exist in a wide class of fractional-order dynamical systems. In fact, many fractional-order analogues of important theoretical results from the classical integer-order dynamical systems theory are still open questions (such as the Hopf bifurcation theorem for fractional-order systems or the stability analysis of time-delayed fractional-order systems (Kaslik and Sivasundaram, 2014)). Therefore, the theoretical analysis of fractional-order models arising from different real world problems has to be done with care.

In fractional-order neural network models, the common capacitor from the continuous-time integer-order recurrent neural networks is replaced by a generalized capacitor, called fractance (Nakagawa and Sorimachi, 1992; Fra, 2007). In many engineering applications, there is a need for lossy capacitors with prescribed losses, in order to accomplish analog fractional calculus operations within a single device. A review of circuit theory approaches aimed at creating fractional-order capacitors (fractance devices) has been presented by Elwakil (2010).

The fractional-order formulation of artificial neural network models is also justified by research results concerning biological neurons. For example, Lundstrom et al. (2008) concluded that fractional differentiation provides neurons with a fundamental and general computation ability that can contribute to efficient information processing, stimulus anticipation and frequency-independent phase shifts of oscillatory neuronal firing. The results reported by Anastasio (1994) suggest that the resulting net output of 
motor and premotor neurons can be described as fractional differentiation relative to eye position.

Fractional-order real-valued artificial neural networks have been in the spotlight since the year 2000, starting with the pioneering works of Arena et al. (2000); Matsuzaki and Nakagawa (2003); Petras (2006); Boroomand and Menhaj (2009), which mainly report on results of numerical simulations, especially on the numerical evidence of limit cycles and chaotic phenomena. Several early papers also discuss chaotic synchronization in fractional-order neural networks (Zhu et al., 2008; Zhou et al., 2008, 2009). The first papers devoted to the theoretical stability analysis and Hopf bifurcations of fractionalorder neural networks of Hopfield type (Kaslik and Sivasundaram, 2011, $2012 \mathrm{~b}$ ) also describe potential routes towards the onset of chaotic behavior when the fractional order of the system increases. The numerical examples presented in these papers unveiled highly complex dynamical behavior in real-valued fractional-order neural networks, such as the co-existence of strange attractors with several asymptotically stable steady states and limit cycles. Moreover, in the last five years, a large number of papers have been published in this field, focusing on theoretical topics such as global MittagLeffler stability and synchronization (Chen et al., 2014), undamped oscillations generated by Hopf bifurcations (Xiao et al., 2015), dynamics of delayed fractional-order neural networks (Chen et al., 2013; Wang et al., 2014a), etc.

However, since many interesting applications of neural networks involve complex signals, such as pattern recognition and classification, intelligent image processing, nonlinear filtering, brain-computer interfaces, time series prediction, robotics and bioinformatics, the investigation of complexvalued neural networks (CVNNs) is an essential extension of the analysis of real-valued neural networks. Complex-valued neural networks (CVNNs) are networks that use complex-valued variables and parameters, successfully dealing in this manner with complex-valued information.

CVNNs are exceptionally rich in diversity and because they are very compatible with wave phenomena, they are appropriate for the processes associated with complex altitude (e.g. interferometric radar systems). Usually, propagation and interference of electromagnetic waves are expressed by the magnitude of transmission and reflection, phase progression and retardation, superposition of fields and so on, phenomena which might be naturally expressed by the use of complex numbers (Hirose, 2006). Correspondingly, these phenomena are correlated with basic processes in the CVNNs, for instance weighting at synaptic connections, i.e. multiplications in amplitude and shifts in phase, and summation of the weighted inputs. CVNNs provide systems with appropriate information representations in many other fields, 
most of them being related to wave phenomena, for example: measurements using waves such as radar image processing, active antennas in electromagnetism, analysis and synthesis in voice processing, learning electronic-wave devices etc.

Several important research directions have arisen concerning CVNNs, such as: the formal generalization of commonly used algorithms to the complex-valued case, the use of original complex-valued activation functions that can increase significantly the neuron and network functionality and the development of quaternion neurons and neural networks (Hirose, 2009).

To the best of our knowledge, there are only a few research papers dedicated to the investigation of fractional-order complex-valued neural networks, published very recently. Sufficient conditions for finite-time stability (Rakkiyappan et al., 2014), uniform asymptotic stability (Rakkivappan et al., 2015a),$O\left(t^{-\alpha}\right)$-stability and global asymptotical periodicity (Rakkivappan et al., 2016) have been obtain for fractional-order complex-valued neural networks with time-delays. In Rakkivappan et al. (2015b), linearization techniques have been used to obtain sufficient conditions for the asymptotic stability of the equilibrium states of fractional-order complex-valued neural networks with time-delays. Necessary conditions for the synchronization of fractionalorder complex-valued neural networks with time delays has been obtained by Bao et al. (2016). Results concerning bifurcation phenomena in delayed fractional complex-valued neural networks have been recently reported by Huang et al. (2017).

It is important to emphasize that, to the best of our knowledge, at this moment, there are no known results concerning Hopf bifurcations in fractional-order complex-valued neural networks, and therefore, this constitutes one of the aims of this paper. Moreover, this paper is devoted to the theoretical stability analysis of fractional-order complex-valued neural networks of Hopfield type, extending the results presented by Kaslik and Sivasundaram (2011, 2012b) for fractional-order real-valued neural networks. Two special connectivity types will be discussed in detail: networks with hub and ring structures, respectively. These simplified connectivity structures are studied to gain insight into the mechanisms underlying the behavior of recurrent networks with more complicated connectivity. Studying patterns of interconnections, called "network motifs" (Milo et al., 2002), occurring in neural networks is fundamental to understanding the dynamic behavior of the whole network.

The paper is structured as follows: in section 2, a few preliminaries are included about fractional-order differential systems, while in section 3, a basic results are described regarding complex-valued fractional-order neural 
networks of Hopfield type. In section 4, a detailed stability and Hopf bifurcation analysis is undertaken for fractional-order Hopfield neural networks, concentrating on the two previously mentioned connectivity structures (hub and ring). In each case, numerical examples are also presented. Concluding remarks are included in section 5 .

\section{Preliminaries on fractional-order differential systems}

The fractional derivate employed in this paper is the Caputo derivative, which is widely considered more applicable to real world problems, as it only requires initial conditions given in terms of integer-order derivatives, representing well-understood features of physical situations (Podlubny, 1999).

Definition 1. For a continuous function $f$, with $f^{\prime} \in L_{\text {loc }}^{1}\left(\mathbb{R}^{+}\right)$, the Caputo fractional-order derivative of order $q \in(0,1)$ of $f$ is defined by

$$
{ }^{c} D^{q} f(t)=\frac{1}{\Gamma(1-q)} \int_{0}^{t}(t-s)^{-q} f^{\prime}(s) d s,
$$

where the gamma function is defined, as usual, as:

$$
\Gamma(z)=\int_{0}^{\infty} e^{-t} t^{z-1} d t .
$$

Remarkable scientific books which provide the main theoretical tools for the qualitative analysis of fractional-order dynamical systems, and at the same time, show the interconnection as well as the contrast between classical differential equations and fractional differential equations, are the works of Podlubny (1999); Kilbas et al. (2006); Lakshmikantham et al. (2009).

The following stability result holds for linear autonomous fractionalorder systems (see Matignon (1996); Sabatier and Farges (2012)):

Theorem 1. The linear autonomous system

$$
{ }^{c} D^{q} \mathbf{x}=A \mathbf{x}
$$

where $A \in \mathbb{R}^{n \times n}$ and $q \in(0,1)$ is asymptotically stable if and only if

$$
|\arg (\lambda)|>\frac{q \pi}{2} \quad \forall \lambda \in \sigma(A)
$$

or equivalently, if and only if

$$
|\Im(\lambda)|>\Re(\lambda) \tan \frac{q \pi}{2} \quad \forall \lambda \in \sigma(A)
$$


where $\sigma(A)$ denotes the spectrum of the matrix $A$ (i.e. the set of all eigenvalues), $\Re(\lambda)$ and $\Im(\lambda)$ denote respectively, the real and imaginary part of $\lambda$.

Remark 1. The integer order linear system $\dot{\mathbf{x}}=A \mathbf{x}$ is asymptotically stable if and only if $\Re(\lambda)<0$ for any $\lambda \in \sigma(A)$. Moreover, it is easy to see that inequality (3) is satisfied for any $\lambda \in \sigma(A)$ with $\Re(\lambda)<0$ and $q \in(0,1)$.

Therefore, if the integer order linear system $\dot{\mathbf{x}}=A \mathbf{x}$ is asymptotically stable, it follows that the fractional order system (11) is also asymptotically stable, for any $q \in(0,1)$.

The converse of the above statement is generally not true. However, in the special case when all the eigenvalues of the matrix $A$ are real (for example if $A$ is a symmetric matrix), the inequality (3) is satisfied if and only if all the eigenvalues of $A$ are strictly negative. We conclude that in this case, asymptotic stability of the fractional-order linear system (11) is equivalent to the asymptotic stability of the integer order system $\dot{\mathbf{x}}=A \mathbf{x}$.

In general, for $0<q_{1}<q_{2} \leq 1$, if the linear system ${ }^{c} D^{q_{2}} \mathbf{x}=A \mathbf{x}$ is asymptotically stable, from Theorem 11 it follows that

$$
|\arg (\lambda)|>\frac{q_{2} \pi}{2}>\frac{q_{1} \pi}{2}, \quad \forall \lambda \in \sigma(A)
$$

and hence, the linear system ${ }^{c} D^{q_{1}} \mathbf{x}=A \mathbf{x}$ is also asymptotically stable.

\section{Complex-valued fractional-order HNNs}

A real-valued fractional-order neural network model of Hopfield type (FHNN) with Caputo-type derivatives, introduced by Kaslik and Sivasundaram (2011, 2012b), is represented by the following system

$$
{ }^{c} D^{q} x_{k}(t)=-a_{k} x_{k}(t)+\sum_{j=1}^{n} T_{k j} g_{j}\left(x_{j}(t)\right)+I_{k}, \quad \forall k=\overline{1, n}, \forall t>0
$$

where $q \in(0,1), a_{k}>0$ are the self-regulating parameters of the neurons, $T=\left(T_{k j}\right)_{n \times n} \in \mathbb{R}^{n \times n}$ is the interconnection matrix, $g_{k}: \mathbb{R} \rightarrow \mathbb{R}$ are the neuron input-output activation functions and $I_{k} \in \mathbb{R}$ denote the external inputs.

In this paper, we generalize the previously considered model (44), introducing the following complex-valued neural network model with Caputo fractional-order derivatives, described by the following system:

$$
{ }^{c} D^{q} z_{k}(t)=-a_{k} z_{k}(t)+\sum_{j=1}^{n} T_{k j} g_{j}\left(z_{j}(t)\right)+I_{k}, \quad \forall k=\overline{1, n}, \forall t>0
$$


where $z_{k}: \mathbb{R}^{+} \rightarrow \mathbb{C}$ are the complex state variables, $a_{k}>0$ are the selfregulating parameters of the neurons, $T=\left(T_{k j}\right)_{n \times n} \in \mathbb{C}^{n \times n}$, is the complex interconnection matrix, $g_{k}: \mathbb{C} \rightarrow \mathbb{C}$ are complex-valued activation functions and $I_{k} \in \mathbb{C}$ represent the complex external inputs.

Several types of activation functions which are often used in complexvalued neural networks are described by Kuroe et al. (2003). In particular, Georgiou and Koutsougeras (1992) describe the properties of the following complex activation function

$$
g(z)=\frac{z}{c_{1}+c_{2}|z|} \quad \text { with } c_{1}, c_{2}>0 .
$$

which proves to be useful in many practical applications.

Denoting $\mathbf{z}(t)=\left(z_{1}(t), z_{2}(t), \ldots, z_{n}(t)\right)^{T}, A=\operatorname{diag}\left(a_{1}, a_{2}, \ldots, a_{n}\right) \in \mathbb{R}^{n \times n}$, $\mathbf{g}(\mathbf{z})=\left(g_{1}\left(z_{1}\right), g_{2}\left(z_{2}\right), \ldots, g_{n}\left(z_{n}\right)\right)^{T}$ and $\mathbf{I}=\left(I_{1}, I_{2}, \ldots, I_{n}\right)^{T} \in \mathbb{C}^{n}$, the system (5) can be written in the following vector form:

$$
{ }^{c} D^{q} \mathbf{z}(t)=-A \mathbf{z}(t)+T \mathbf{g}(\mathbf{z}(t))+\mathbf{I} .
$$

Considering the real and the imaginary parts of the complex state vector, interconnection matrix, input vector and activation functions respectively, we denote

$$
\begin{aligned}
& \mathbf{z}(t)=\mathbf{x}(t)+i \mathbf{y}(t), \text { where } \mathbf{x}, \mathbf{y}: \mathbb{R}^{+} \rightarrow \mathbb{R}^{n}, \\
& T=T^{R}+i T^{I}, \text { where } T^{R}, T^{I} \in \mathbb{R}^{n \times n}, \\
& \mathbf{I}=\mathbf{I}^{R}+i \mathbf{I}^{I}, \text { where } \mathbf{I}^{R}, \mathbf{I}^{I} \in \mathbb{R}^{n}, \\
& \mathbf{g}(\mathbf{z})=\mathbf{g}(\mathbf{x}+i \mathbf{y})=\mathbf{g}^{R}(\mathbf{x}, \mathbf{y})+i \mathbf{g}^{I}(\mathbf{x}, \mathbf{y}) \\
& \text { with }\left\{\begin{array}{c}
\mathbf{g}^{R}(\mathbf{x}, \mathbf{y})=\left(g_{1}^{R}\left(x_{1}, y_{1}\right), g_{2}^{R}\left(x_{2}, y_{2}\right), \ldots, g_{n}^{R}\left(x_{n}, y_{n}\right)\right)^{T} \\
\mathbf{g}^{I}(\mathbf{x}, \mathbf{y})=\left(g_{1}^{I}\left(x_{1}, y_{1}\right), g_{2}^{I}\left(x_{2}, y_{2}\right), \ldots, g_{n}^{I}\left(x_{n}, y_{n}\right)\right)^{T}
\end{array}\right.
\end{aligned}
$$

With the above notations, system (7) becomes

$$
{ }^{c} D^{q}(\mathbf{x}+i \mathbf{y})=-A(\mathbf{x}+i \mathbf{y})+\left(T^{R}+i T^{I}\right)\left(\mathbf{g}^{R}(\mathbf{x}, \mathbf{y})+i \mathbf{g}^{I}(\mathbf{x}, \mathbf{y})\right)+\mathbf{I}^{R}+i \mathbf{I}^{I} .
$$

which is equivalent to the following real-valued fractional-order system:

$$
\left\{\begin{array}{l}
{ }^{c} D^{q} \mathbf{x}(t)=-A \mathbf{x}+T^{R} \mathbf{g}^{R}(\mathbf{x}, \mathbf{y})-T^{I} \mathbf{g}^{I}(\mathbf{x}, \mathbf{y})+\mathbf{I}^{R} \\
{ }^{c} D^{q} \mathbf{y}(t)=-A \mathbf{y}+T^{R} \mathbf{g}^{I}(\mathbf{x}, \mathbf{y})+T^{I} \mathbf{g}^{R}(\mathbf{x}, \mathbf{y})+\mathbf{I}^{I}
\end{array}\right.
$$


Denoting

$$
\begin{aligned}
\mathbf{u}(t) & =(\mathbf{x}(t), \mathbf{y}(t))^{T} \in \mathbb{R}^{2 n} \\
\tilde{A} & =\left(\begin{array}{cc}
A & 0 \\
0 & A
\end{array}\right)=\operatorname{diag}\left(a_{1}, a_{2}, \ldots, a_{n}, a_{1}, a_{2}, \ldots, a_{n}\right) \in \mathbb{R}^{2 n \times 2 n}, \\
\tilde{T} & =\left(\begin{array}{cc}
T^{R} & -T^{I} \\
T^{I} & T^{R}
\end{array}\right) \in \mathbb{R}^{2 n \times 2 n}, \\
\tilde{\mathbf{g}}(\mathbf{u}) & =\tilde{\mathbf{g}}\left((\mathbf{x}, \mathbf{y})^{T}\right)=\left(\mathbf{g}^{R}(\mathbf{x}, \mathbf{y}), \mathbf{g}^{I}(\mathbf{x}, \mathbf{y})\right)^{T}= \\
& =\left(g_{1}^{R}\left(x_{1}, y_{1}\right), . ., g_{n}^{R}\left(x_{n}, y_{n}\right), g_{1}^{I}\left(x_{1}, y_{1}\right), . ., g_{n}^{I}\left(x_{n}, y_{n}\right)\right)^{T} \in \mathbb{R}^{2 n} \\
\tilde{\mathbf{I}} & =\left(\mathbf{I}^{R}, \mathbf{I}^{I}\right)^{T} \in \mathbb{R}^{2 n},
\end{aligned}
$$

the $n$-dimensional complex system (17) is then equivalent to the $2 n$-dimensional fractional-order real-valued system

$$
{ }^{c} D^{q} \mathbf{u}(t)=-\tilde{A} \mathbf{u}(t)+\tilde{T} \tilde{\mathbf{g}}(\mathbf{u}(t))+\tilde{\mathbf{I}} .
$$

It has to be emphasized that system (9) is equivalent to a real-valued bidirectional associative memory (BAM) network if and only if

$$
g_{j}^{R}\left(x_{j}, y_{j}\right)=g_{j}^{R}\left(y_{j}\right) \quad \text { and } \quad g_{j}^{I}\left(x_{j}, y_{j}\right)=g_{j}^{I}\left(x_{j}\right), \quad \text { for any } j=\overline{1, n} \text {, }
$$

i.e., the real part of the complex activation function $g_{j}$ depends only on the imaginary part $y_{j}$ of the state variable, and the imaginary part of the of $g_{j}$ depends only on the real part $x_{j}$ of the state variable.

\section{Stability and bifurcations}

In the following, let us consider $\mathbf{z}^{*}=\mathbf{x}^{*}+i \mathbf{y}^{*} \in \mathbb{C}^{n}$ an equilibrium state of the complex-valued fractional-order neural network (7):

$$
-A \mathbf{z}^{*}+T \mathbf{g}\left(\mathbf{z}^{*}\right)+\mathbf{I}=0 .
$$

Equivalently, $\mathbf{u}^{*}=\left(\mathbf{x}^{*}, \mathbf{y}^{*}\right)^{T}$ is a steady state of the real system (9), i.e. a solution of

$$
-\tilde{A} \mathbf{u}^{*}+\tilde{T} \tilde{\mathbf{g}}\left(\mathbf{u}^{*}\right)+\tilde{\mathbf{I}}=0 .
$$

Obviously, $\mathbf{z}^{*} \in \mathbb{C}^{n}$ is a steady state of the system (77) with the fractional order $q \in(0,1)$ if and only if it is an equilibrium state of the corresponding integer order system (i.e. for $q=1$ ). Therefore, the same results hold for the existence, uniqueness or multiplicity of equilibrium states of fractional-order 
neural networks, as in the case of integer-order neural networks. However, the conditions for the asymptotic stability of an equilibrium state $\mathbf{z}^{*} \in \mathbb{C}^{n}$ are in general more strict in the case of the corresponding integer-order system.

With the aim of studying the stability of the equilibrium state $\mathbf{z}^{*}$ in the framework of the fractional-order system (7), or equivalently, the stability of the equilibrium state $\mathbf{u}^{*}$ of system (9), we rely on the linearization theorem recently proved by $\mathrm{Li}$ and $\mathrm{Ma}(2013)$. This linearization theorem is an analogue of the classical Hartman-Grobman theorem for the case of integerorder dynamical systems. For a rigorous application of this linearization theorem, we have to require that the function $\tilde{\mathrm{g}}$ is of class $C^{1}$ (continuously differentiable) on a neighborhood of the steady state $\mathbf{u}^{*} \in \mathbb{R}^{2 n}$. Using the notations $g_{k}: \mathbb{C} \rightarrow \mathbb{C}$, with $g_{k}(z)=g_{k}(x+i y)=g_{k}^{R}(x, y)+i g_{k}^{I}(x, y)$, for the complex activation functions, this is equivalent to the following assumption:

(A) $g_{k}^{R}$ and $g_{k}^{I}$ are of class $C^{1}$ in a neighborhood of $\left(x_{k}^{*}, y_{k}^{*}\right) \in \mathbb{R}^{2}, \forall k=\overline{1, n}$.

Due to the fact that the activation functions $g_{k}$ are usually assumed to be bounded, we cannot require them to be holomorphic on the whole complex plane $\mathbb{C}$ (i.e. entire functions). The reason is that, according to Liouville's theorem, every bounded entire function must be constant. Therefore, for simplicity, we only assume the following:

$\left(\mathbf{A}_{2}\right) \quad g_{k}$ are complex-differentiable at the point $z_{k}^{*}=x_{k}^{*}+i y_{k}^{*}, \forall k=\overline{1, n}$.

Hence, the Cauchy-Riemann conditions are satisfied:

$$
\left\{\begin{aligned}
\frac{\partial g_{k}^{R}}{\partial x}\left(x_{k}^{*}, y_{k}^{*}\right) & =\frac{\partial g_{k}^{I}}{\partial y}\left(x_{k}^{*}, y_{k}^{*}\right), \\
\frac{\partial g_{k}^{R}}{\partial y}\left(x_{k}^{*}, y_{k}^{*}\right) & =-\frac{\partial g_{k}^{I}}{\partial x}\left(x_{k}^{*}, y_{k}^{*}\right) .
\end{aligned}\right.
$$

Remark 2. The activation function $g(z)$ given by (6) satisfies assumption $\left(A_{1}\right)$. In fact, the real and imaginary parts $g^{R}$ and $g^{I}$ are of class $C^{1}$ on $\mathbb{R}^{2}$. A detailed analysis has been performed by Georgiou and Koutsougeras (1992). Moreover, the function $g(z)$ is complex-differentiable at 0 and the complex derivative is $g^{\prime}(0)=1$.

Based on the previous assumptions and the Hartman-Grobman-type linearization theorem ( $\mathrm{Li}$ and $\mathrm{Ma}, 2013)$, the asymptotic stability of the steady state $\mathbf{u}^{*} \in \mathbb{R}^{2 n}$ of the fractional-order system (9) is determined by the eigenvalues of the Jacobian matrix

$$
\tilde{J}\left(\mathbf{u}^{*}\right)=-\tilde{A}+\tilde{T} D \tilde{\mathbf{g}}\left(\mathbf{u}^{*}\right),
$$


where

$$
D \tilde{\mathbf{g}}\left(\mathbf{u}^{*}\right)=\left(\begin{array}{cc}
D \mathbf{g}_{x}^{R}\left(\mathbf{u}^{*}\right) & D \mathbf{g}_{y}^{R}\left(\mathbf{u}^{*}\right) \\
-D \mathbf{g}_{y}^{R}\left(\mathbf{u}^{*}\right) & D \mathbf{g}_{x}^{R}\left(\mathbf{u}^{*}\right)
\end{array}\right)
$$

is a block matrix obtained using conditions (10):

$$
\begin{aligned}
& D \mathbf{g}_{x}^{R}\left(\mathbf{u}^{*}\right)=\operatorname{diag}\left(\frac{\partial g_{1}^{R}}{\partial x_{1}}\left(x_{1}^{*}, y_{1}^{*}\right), \frac{\partial g_{2}^{R}}{\partial x_{2}}\left(x_{2}^{*}, y_{2}^{*}\right), \ldots, \frac{\partial g_{n}^{R}}{\partial x_{n}}\left(x_{n}^{*}, y_{n}^{*}\right)\right) \\
& D \mathbf{g}_{y}^{R}\left(\mathbf{u}^{*}\right)=\operatorname{diag}\left(\frac{\partial g_{1}^{R}}{\partial y_{1}}\left(x_{1}^{*}, y_{1}^{*}\right), \frac{\partial g_{2}^{R}}{\partial y_{2}}\left(x_{2}^{*}, y_{2}^{*}\right), \ldots, \frac{\partial g_{n}^{R}}{\partial y_{n}}\left(x_{n}^{*}, y_{n}^{*}\right)\right) .
\end{aligned}
$$

Furthermore, the Jacobian matrix becomes

$$
\tilde{J}\left(\mathbf{u}^{*}\right)=-\tilde{A}+\tilde{T} D \tilde{\mathbf{g}}\left(\mathbf{u}^{*}\right)=-\left(\begin{array}{cc}
A & 0 \\
0 & A
\end{array}\right)+\left(\begin{array}{cc}
T^{R} & -T^{I} \\
T^{I} & T^{R}
\end{array}\right)\left(\begin{array}{cc}
D_{1} & -D_{2} \\
D_{2} & D_{1}
\end{array}\right),
$$

where $A=\operatorname{diag}\left(a_{1}, . ., a_{n}\right), D_{1}=D \mathbf{g}_{x}^{R}\left(\mathbf{u}^{*}\right), D_{2}=-D \mathbf{g}_{y}^{R}\left(\mathbf{u}^{*}\right)$ are real diagonal matrices.

In order to simplify the computations, we use the following notations

$$
\begin{aligned}
U & =-A+T^{R} D_{1}-T^{I} D_{2}, \\
V & =T^{R} D_{2}+T^{I} D_{1} .
\end{aligned}
$$

Consequently, the Jacobian matrix (11) becomes

$$
\tilde{J}\left(\mathbf{u}^{*}\right)=\left(\begin{array}{cc}
U & -V \\
V & U
\end{array}\right)
$$

The characteristic polynomial of the Jacobian matrix $\tilde{J}\left(\mathbf{u}^{*}\right)$ is

$$
\begin{array}{rlr}
P(\lambda) & =\operatorname{det}\left(\tilde{J}\left(\mathbf{u}^{*}\right)-\lambda I_{2 n}\right) & \\
& =\operatorname{det}\left(\begin{array}{cc}
U-\lambda I_{n} & -V \\
V & U-\lambda I_{n}
\end{array}\right) & \left(\mathrm{Row}_{1}+i \cdot \mathrm{Row}_{2}\right) \\
& =\operatorname{det}\left(\begin{array}{cc}
U-\lambda I_{n}+i V & -V+i U-i \lambda I_{n} \\
V &
\end{array}\right) & \left(\mathrm{Col}_{2}-i \cdot \mathrm{Col}_{1}\right) \\
& =\operatorname{det}\left(\begin{array}{cc}
U-\lambda I_{n}+i V & O_{n} \\
V & U-\lambda I_{n}-i V
\end{array}\right) \\
& =\operatorname{det}\left(U+i V-\lambda I_{n}\right) \cdot \operatorname{det}\left(U-i V-\lambda I_{n}\right) .
\end{array}
$$

Consequently, the roots of the characteristic polynomial $P(\lambda)$ are either the eigenvalues of the matrix $U+i V$, or the eigenvalues of the matrix $U-i V=$ 
$\overline{U+i V}$. In the following, let us denote $M=U+i V$ and $\sigma(M)$ the spectrum of $M$. Then, $\sigma\left(\tilde{J}\left(u^{*}\right)\right)=\sigma(M) \cup \sigma(\bar{M})=\sigma(M) \cup \overline{\sigma(M)}$, so we only need to determine the eigenvalues of the matrix $M$.

We can easily see that

$$
\begin{aligned}
M & =-A+T^{R} D_{1}-T^{I} D_{2}+i\left(T^{R} D_{2}+T^{I} D_{1}\right)= \\
& =-A+\left(T^{R}+i T^{I}\right)\left(D_{1}+i D_{2}\right)= \\
& =-A+T \mathbf{g}^{\prime}\left(\mathbf{z}^{*}\right),
\end{aligned}
$$

where $\mathbf{g}^{\prime}\left(\mathbf{z}^{*}\right)$ is the complex Jacobian matrix of the function $\mathbf{g}$ at the equilibrium state $\mathbf{z}^{*}$ :

$$
\mathbf{g}^{\prime}\left(\mathbf{z}^{*}\right)=\operatorname{diag}\left(g_{1}^{\prime}\left(z_{1}^{*}\right), g_{2}^{\prime}\left(z_{2}^{*}\right), \ldots, g_{n}^{\prime}\left(z_{n}^{*}\right)\right) .
$$

Based on the above reasoning and Theorem 1, we conclude:

Proposition 1. If assumptions $\left(A_{1}\right)$ and $\left(A_{2}\right)$ hold, the equilibrium state $\mathbf{z}^{*}$ of system (17) is asymptotically stable if and only if all the eigenvalues of the complex-valued matrix $-A+T \mathbf{g}^{\prime}\left(\mathbf{z}^{*}\right)$ satisfy $|\arg (\lambda)|>\frac{q \pi}{2}$.

In the following section, the stability and bifurcation properties will be investigated in the case of a special network structure, called the hub structure.

\subsection{Complex-valued FHNN with hub structure}

In scale-free networks (Barabási and Albert, 1999), some nodes, called "hubs", have many more connections than other nodes. In fact, the network as a whole has a power-law distribution of the number of links connecting to a node (at least asymptotically). In this type of networks, the existence of hub structures is a common feature, playing a fundamental role in defining the connectivity of the scale-free networks and in characterizing their dynamical behavior. The dynamics of hubs for integer-order real-valued neural networks was studied by Kitajima and Kurths (2009). Real-valued fractional-order neural networks with hub structure have been investigated by Kaslik and Sivasundaram (2012b) in the non-delayed case and by Wang et al. (2014b) in the delayed case.

In the following, we consider the complex-valued fractional-order neural network of $n \geq 3$ neurons with hub structure

$$
\left\{\begin{array}{l}
{ }^{c} D^{q} z_{1}(t)=-a z_{1}(t)+\sum_{j=1}^{n} T_{1 j} g_{j}\left(z_{j}(t)\right)+I_{1} \\
{ }^{c} D^{q} z_{j}(t)=-b_{j} z_{j}(t)+T_{j 1} g_{1}\left(z_{1}(t)\right)+T_{j j} g_{j}\left(z_{j}(t)\right)+I_{j}, \quad \forall j=\overline{2, n}
\end{array}\right.
$$


where $a, b_{j}>0, T_{j k} \in \mathbb{C}, \forall j, k=\overline{1, n}$. Here, the first neuron (called the central neuron) is the center of the hub, and all the other $(n-1)$ neurons (called peripheral neurons) are connected directly only to the central neuron and to themselves (self-connections are present). The interconnection matrix of this neural network is

$$
T=\left(\begin{array}{ccccc}
T_{11} & T_{12} & T_{13} & \ldots & T_{1 n} \\
T_{21} & T_{22} & 0 & \ldots & 0 \\
T_{31} & 0 & T_{33} & \ldots & 0 \\
\ldots & \ldots & \ldots & \ldots & \ldots \\
T_{n 1} & 0 & 0 & \ldots & T_{n n}
\end{array}\right) \in \mathbb{C}^{n \times n}
$$

Let $\mathbf{z}^{*}=\left(z_{1}^{*}, z_{2}^{*}, \ldots, z_{n}^{*}\right)^{T}$ an equilibrium of the system (13) and, for simplicity, we denote

$$
-a+T_{11} g_{1}^{\prime}\left(z_{1}^{*}\right)=\alpha
$$

and assume that

$$
-b_{j}+T_{j j} g_{j}^{\prime}\left(z_{j}^{*}\right)=\beta, \quad \forall j=\overline{2, n} .
$$

For example, if the peripheral neurons are identical, assumption (15) holds always true.

Consequently, from (12), (14) and (15) the Jacobian matrix of the system (13) is

$$
J\left(\mathbf{z}^{*}\right)=\left(\begin{array}{ccccc}
\alpha & T_{12} g_{2}^{\prime}\left(z_{2}^{*}\right) & T_{13} g_{3}^{\prime}\left(z_{3}^{*}\right) & \ldots & T_{1 n} g_{n}^{\prime}\left(z_{n}^{*}\right) \\
T_{21} g_{1}^{\prime}\left(z_{1}^{*}\right) & \beta & 0 & \ldots & 0 \\
T_{31} g_{1}^{\prime}\left(z_{1}^{*}\right) & 0 & \beta & \ldots & 0 \\
\ldots & \ldots & \ldots & \ldots & \ldots \\
T_{n 1} g_{1}^{\prime}\left(z_{1}^{*}\right) & 0 & 0 & \ldots & \beta
\end{array}\right) .
$$

Using the notation

$$
\gamma=\sum_{k=2}^{n} T_{1 k} T_{k 1} g_{1}^{\prime}\left(z_{1}^{*}\right) g_{k}^{\prime}\left(z_{k}^{*}\right),
$$

we obtain the characteristic equation

$$
(\lambda-\beta)^{n-2}\left(\lambda^{2}-(\alpha+\beta) \lambda+\alpha \beta-\gamma\right)=0,
$$

with $\alpha, \beta, \gamma \in \mathbb{C}$ defined above depending on the equilibrium $\mathbf{z}^{*}$ and the system parameters $a, b_{i}, T_{i j}$ of (13). 
Remark 3. Let $\lambda_{1}, \lambda_{2}$ denote the roots of the second order polynomial with complex coefficients

$$
P(\lambda)=\lambda^{2}-(\alpha+\beta) \lambda+\alpha \beta-\gamma
$$

If $n \geq 3$, it is easy to see that $\lambda_{0}=\beta$ is a root of the characteristic equation (17), with the order of multiplicity $(n-2)$. Therefore:

$$
\sigma\left(J\left(\mathbf{z}^{*}\right)\right)=\left\{\beta, \lambda_{1}, \lambda_{2}\right\} .
$$

On the other hand, in the particular case $n=2$, we simply obtain

$$
\sigma\left(J\left(\mathbf{z}^{*}\right)\right)=\left\{\lambda_{1}, \lambda_{2}\right\} .
$$

In the following, with the aim of analyzing the stability of the steady state $\mathbf{z}^{*}$ using the characteristic equation (17) and Theorem 1, we will concentrate our attention on the roots of the second-order polynomial $P(\lambda)$ defined in Remark 3. We denote:

$$
\begin{array}{ll}
\rho_{1}=|\alpha+\beta| & \theta_{1}=\operatorname{Arg}(\alpha+\beta) \in(-\pi, \pi] \\
\rho_{2}=|\alpha \beta-\gamma|>0 & \theta_{2}=\operatorname{Arg}(\alpha \beta-\gamma) \in(-\pi, \pi]
\end{array}
$$

and

$$
\begin{aligned}
& A=\theta_{1}-\frac{\theta_{2}}{2} \in\left(-\frac{3 \pi}{2}, \frac{3 \pi}{2}\right) \\
& B=\frac{1}{2} \arccos \left\{\frac{1}{2}\left[\frac{\rho_{1}^{2}}{2 \rho_{2}}-\sqrt{\frac{\rho_{1}^{4}}{4 \rho_{2}^{2}}+4-2 \frac{\rho_{1}^{2}}{\rho_{2}} \cos (2 A)}\right]\right\} \in\left[0, \frac{\pi}{2}\right] .
\end{aligned}
$$

The following result can be proved using standard mathematical tools:

Lemma 1. Let $\phi_{1}, \phi_{2} \in(-\pi, \pi]$ denote the principal arguments of the roots of the polynomial $P(\lambda)$. The following cases occur:

(a) If $\cos (A) \geq 0$, then $\phi_{1,2}=\frac{\theta_{2}}{2} \pm B$;

(b) If $\cos (A)<0$, then

(b.1) if $\theta_{2} \leq-2 B$ then $\phi_{1,2}=\frac{\theta_{2}}{2}+\pi \pm B$;

(b.2) if $\theta_{2} \in(-2 B, 2 B]$ then $\phi_{1,2}=\frac{\theta_{2}}{2} \pm(\pi-B)$; 
(b.3) if $\theta_{2}>2 B$ then $\phi_{1,2}=\frac{\theta_{2}}{2}-\pi \pm B$;

Proof. See Appendix.

Based on Remark 3 and Lemma 1, we obtain the following result regarding the asymptotic stability of the equilibrium point $\mathbf{z}^{*}$.

Proposition 2. Let $q^{* *}$ be defined as follows:

$q^{* *}= \begin{cases}\frac{1}{\pi} \min \left\{\left|\theta_{2}-2 B\right|,\left|\theta_{2}+2 B\right|\right\}, & \text { if } \cos (A) \geq 0 \\ \frac{1}{\pi} \min \left\{\left|\theta_{2}+2 \pi-2 B\right|,\left|\theta_{2}+2 \pi+2 B\right|\right\}, & \text { if } \cos (A)<0 \text { and } \theta_{2} \leq-2 B \\ \frac{1}{\pi} \min \left\{\left|\theta_{2}+2 \pi-2 B\right|,\left|\theta_{2}-2 \pi+2 B\right|\right\}, & \text { if } \cos (A)<0 \text { and } \theta_{2} \in(-2 B, 2 B] \\ \frac{1}{\pi} \min \left\{\left|\theta_{2}-2 \pi+2 B\right|,\left|\theta_{2}-2 \pi-2 B\right|\right\}, & \text { if } \cos (A)<0 \text { and } \theta_{2}>2 B\end{cases}$

The steady state $\mathbf{z}^{*}$ of the system (13) is asymptotically stable if and only if $q \in\left(0, q^{*}\right) \cap(0,1)$, where

$$
q^{*}= \begin{cases}\min \left\{\frac{2}{\pi}|\operatorname{Arg}(\beta)|, q^{* *}\right\} & , \text { if } n \geq 3 \\ q^{* *} & , \text { if } n=2\end{cases}
$$

Proof. The steady state $\mathbf{z}^{*}$ of the system (13) is asymptotically stable if and only if the arguments of the all the roots of the characteristic equation (17) satisfy inequality (2) from Theorem 1. If $n \geq 3$, with the notations from Remark 3, we know that these roots are $\left\{\beta, \lambda_{1}, \lambda_{2}\right\}$. On the other hand, when $n=2$, the characteristic roots are only $\left\{\lambda_{1}, \lambda_{2}\right\}$. Based on Lemma 1 we obtain the desired result.

Remark 4. The case $n=2$ corresponds to the case of simple hub containing a central neuron and only one peripheral neuron described by a simple twodimensional complex-valued neural network

$$
\left\{\begin{array}{l}
{ }^{c} D^{q} z_{1}(t)=-a z_{1}(t)+T_{11} g_{1}\left(z_{1}(t)\right)+T_{12} g_{2}\left(z_{2}(t)\right)+I_{1} \\
{ }^{c} D^{q} z_{2}(t)=-b z_{2}(t)+T_{21} g_{1}\left(z_{1}(t)\right)+T_{22} g_{2}\left(z_{2}(t)\right)+I_{2}
\end{array}\right.
$$

The stability analysis of the steady state $\mathbf{z}^{*}$ requires the study of the characteristic equation

$$
P(\lambda)=\lambda^{2}-(\alpha+\beta) \lambda+\alpha \beta-\gamma=0
$$


where $\alpha=-a+T_{11} g_{1}^{\prime}\left(z_{1}^{*}\right), \beta=-b+T_{22} g_{2}^{\prime}\left(z_{2}^{*}\right)$ and $\gamma=T_{12} T_{21} g_{1}^{\prime}\left(z_{1}^{*}\right) g_{2}^{\prime}\left(z_{2}^{*}\right)$. Based on Proposition 2, we obtain that the steady state $\mathbf{z}^{*}$ of (19) is asymptotically stable if and only if any $q \in\left(0, q^{* *}\right) \cap(0,1)$.

It is worth mentioning that in the case of fractional-order dynamical systems very few theoretical results are known at this time regarding bifurcation phenomena. El-Saka et al. (2009) attempt to formulate conditions for the occurrence of Hopf bifurcations, based on observations arising from numerical simulations. However, the complete characterization of the Hopf bifurcation in fractional-order dynamical systems, as well as the stability of the resulting limit cycle, are still open questions.

Remark 5. Because a steady state $\mathbf{z}^{*}$ of (13) does not depend on the fractional order $q \in(0,1)$, a good choice for the bifurcation parameter that may be considered in system (13) is the fractional order $q$ itself.

According to Proposition 2, if $q^{*}<1$, the Jacobian matrix $J\left(\mathbf{z}^{*}\right)$ has a pair of complex eigenvalues $\pm \frac{q^{*} \pi}{2}$, and according to El-Saka et al. (2009), this corresponds to a Hopf bifurcation in the fractional-order neural network (13). The steady state $\mathbf{z}^{*}$ of (13) is asymptotically stable if and only if $q \in\left(0, q^{*}\right)$. As $q$ increases and crosses the critical value $q^{*}$, the steady state $\mathbf{z}^{*}$ becomes unstable, and a limit cycle is expected to appear in a neighborhood of $\mathbf{z}^{*}$, due to the Hopf bifurcation phenomenon.

However, if $q^{*} \geq 1$, it follows that $\mathbf{z}^{*}$ is asymptotically stable for any $q \in(0,1)$.

Example 1. The following complex-valued fractional-order neural network of three neurons with hub structure is considered:

$$
\left\{\begin{array}{l}
{ }^{c} D^{q} z_{1}(t)=-z_{1}(t)+(2-5 i) g\left(z_{1}(t)\right)-(2+i) g\left(z_{2}(t)\right)+(2+i) g\left(z_{3}(t)\right) \\
{ }^{c} D^{q} z_{2}(t)=-2 z_{2}(t)+3 g\left(z_{1}(t)\right)+(1+i) g\left(z_{2}(t)\right) \\
{ }^{c} D^{q} z_{3}(t)=-2 z_{3}(t)+(1-i) g\left(x_{1}(t)\right)+(1+i) g\left(z_{3}(t)\right)
\end{array}\right.
$$

where the activation function is $g(z)=\frac{z}{1+|z|}$, with the complex derivative $g^{\prime}(0)=1$. In this neural network, $a=1, b_{2}=b_{3}=2, T_{11}=2-5 i$, $T_{22}=T_{33}=1+i, T_{12}=-2-i, T_{21}=3, T_{13}=2+i$ and $T_{31}=1-i$.

For the steady state $\mathbf{z}^{*}=\mathbf{0}$, the parameters given by the equations (14), (15) and (16) are $\alpha=1-5 i, \beta=-1+i$ and $\gamma=-3-4 i$. We can easily compute the critical value of the fractional order $q$ given by (18), $q^{*}=0.844976$. Hence, the null solution is asymptotically stable if and only if $q \in(0,0.844976)$ and unstable for $q \in(0.844976,1)$. At $q=q^{*}$, a Hopf bifurcation is expected to take place. 

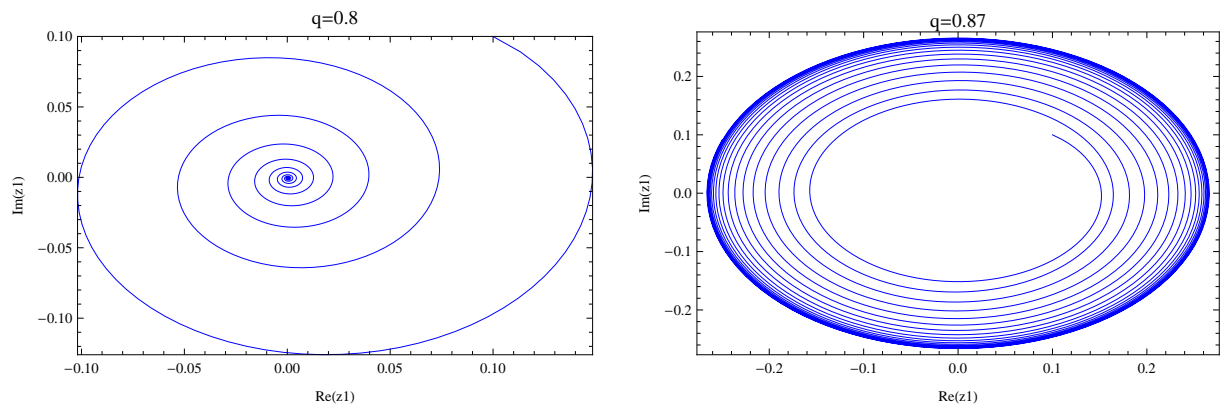

Figure 1: Hopf bifurcation occurring in a neighborhood of the null solution of system (21) at the critical value of the fractional order $q^{*}=0.844976$. The null solution is asymptotically stable for $q=0.8$ (left) and unstable for $q=0.87$ (right). An asymptotically stable limit cycle appears due to the supercritical Hopf bifurcation as $q$ increases above the critical value (right).

Indeed, when the fractional order increases above the critical value $q^{*}=$ 0.844976, numerical simulations show the appearance of an asymptotically stable limit cycle in a neighborhood of the origin (see Fig 1). For all numerical simulations, the generalization of the Adams-Bashforth-Moulton predictorcorrector method has been used, described by Diethelm et al. (2002). Numerical simulations also show the existence of an asymptotically stable limit cycle for any $q \in\left(q^{*}, 1\right)$.

\subsection{Complex-valued FHNN with ring structure}

Another simple connectivity structure frequently considered in neural networks is the ring structure, in which every neuron of the network is only connected to its two closest neighbors. Ring architectures have been found in a variety of neural structures, such as the hippocampus, cerebellum, neocortex, and even in chemistry and electrical engineering. It is a well-known fact that the real cortical connectivity pattern in these network structures is extremely sparse: most connections are between nearby cells, and long-range connections become progressively more infrequent (Hirsch, 1989).

The dynamics of continuous-time neural networks with ring structure, including aspects such as stability, bifurcations, patters of oscillations, nonlinear waves, have been extensively studied by Baldi and Ativa (1994): Campbell et al. (1999); Guo and Huang (2003); Guo (2005); Wei and Jiang (2006); Guo and Huang (2006, 2007b, a); Bungav and Campbell (2007); Lu and Guo (2008). Bifurcation phenomena and chaotic behavior in discrete-time delayed neural rings 
have been discussed by Kaslik and Balint (2009); Kaslik (2009).

Real-valued fractional-order neural networks with ring structure, with and without delays, have been recently analyzed by Kaslik and Sivasundaram (2012b); Wang et al. (2014b, 2015).

Considering, for simplicity, a null external input, we analyze a complexvalued fractional-order neural network with ring structure described by:

$$
\left\{\begin{array}{rl}
{ }^{c} D^{q} z_{1}(t)= & -a_{1} z_{1}(t)+T_{1,1} g_{1}\left(z_{1}(t)\right)+ \\
& +T_{1,2} g_{2}\left(z_{2}(t)\right)+T_{1, n} g_{n}\left(z_{n}(t)\right) \\
{ }^{c} D^{q} z_{j}(t)= & -a_{2} z_{j}(t)+T_{j, j-1} g_{j-1}\left(z_{j-1}(t)\right)+ \\
& +T_{j, j} g_{j}\left(z_{j}(t)\right)+T_{j, j+1} g_{j+1}\left(z_{j+1}(t)\right) \\
{ }^{c} D^{q} z_{n}(t)= & -a_{n} z_{n}(t)+T_{n, 1} g_{1}\left(z_{1}(t)\right)+ \\
& +T_{n, n-1} g_{n-1}\left(z_{n-1}(t)\right)+T_{n, n} g_{n}\left(z_{n}(t)\right)
\end{array} \quad, \forall j=\overline{2, n-1}\right.
$$

where $n \geq 3$. The interconnection matrix of system (22) is

$$
T=\left(\begin{array}{cccccccc}
T_{1,1} & T_{1,2} & 0 & 0 & \ldots & 0 & 0 & T_{1, n} \\
T_{2,1} & T_{2,2} & T_{2,3} & 0 & \ldots & 0 & 0 & 0 \\
0 & T_{3,2} & T_{3,3} & T_{3,4} & \ldots & 0 & 0 & 0 \\
\ldots & \ldots & \ldots & \ldots & \ldots & \ldots & \ldots & \ldots \\
0 & 0 & 0 & 0 & \ldots & T_{n-2, n-2} & T_{n-2, n-1} & 0 \\
0 & 0 & 0 & 0 & \ldots & T_{n-1, n-2} & T_{n-1, n-1} & T_{n-1, n} \\
T_{n, 1} & 0 & 0 & 0 & \ldots & 0 & T_{n, n-1} & T_{n, n} \\
& & & & & & &
\end{array}\right) \in \mathbb{C}^{n \times n}
$$

Let $\mathbf{z}^{*}$ be a steady state of system (22). We will make the following simplifying assumptions:

$$
\begin{aligned}
& -a_{j}+T_{j, j} g_{j}^{\prime}\left(z_{j}^{*}\right)=\alpha, \quad \forall j=\overline{1, n} \\
& T_{j, j+1} g_{j+1}^{\prime}\left(z_{j+1}^{*}\right)=\beta, \quad \forall j=\overline{1, n}
\end{aligned}
$$

and

$$
T_{j, j-1} g_{j-1}^{\prime}\left(z_{j-1}^{*}\right)=\gamma, \quad \forall j=\overline{1, n}
$$

where the complex parameters $\alpha, \beta$ and $\gamma$ depend on the steady state $\mathbf{z}^{*}$ which is being analyzed and the system parameters $a_{j}, T_{j, k}$ of (22).

It is easy to see that these assumptions are satisfied in the case of identical neurons, having the same activation function $g_{j}=g_{0}, j=\overline{1, n}$, the same self-regulating parameters $a_{j}=a_{0}>0, j=\overline{1, n}$, and the connection weights satisfying $T_{j, j}=T_{0}$ (self-connection), $T_{j, j+1}=T_{1}$ (forward connection) and $T_{j, j-1}=T_{2}$ (backward connection), for any $j=\overline{1, n}$. With these 
assumptions, we may consider steady states $\mathbf{z}^{*}$ with equal complex scalar components $z_{1}^{*}=z_{2}^{*}=\ldots=z_{n}^{*}=z^{*}$, such that

$$
-a_{0} z^{*}+\left(T_{0}+T_{1}+T_{2}\right) g_{0}\left(z^{*}\right)=0 .
$$

Obviously, $z^{*}=0$ is a solution of this equation, as the trivial solution is a steady state of (22). Besides the null solution, the equation (27) may have other solutions as well, and therefore, the system (22) may have multiple steady states.

Let us return to the stability analysis of a steady state $\mathbf{z}^{*}$ of system (22). If the assumptions (24), (25) and (26) are fulfilled, we obtain that the Jacobian matrix of system (22) is a circulant matrix of the form

$$
J\left(\mathbf{z}^{*}\right)=\operatorname{circ}(\alpha, \beta, 0, \ldots, 0, \gamma)
$$

The eigenvalues of the complex circulant matrix $J\left(\mathbf{z}^{*}\right)$ are (see for example Grav (2005) and Kra and Simanca (2012)):

$$
\lambda_{p}=\alpha+\beta \omega^{p}+\gamma \bar{\omega}^{p}, \quad \forall p \in\{0,1, . ., n-1\}
$$

where $\omega=\exp \left(\frac{2 \pi i}{n}\right)$.

We easily obtain the following sufficient condition for the asymptotic stability of the steady state $\mathbf{z}^{*}$ :

Proposition 3. If conditions (24), (25) and (26) are satisfied and

$$
\Re(\alpha)+|\beta+\bar{\gamma}|<0,
$$

the steady state $\mathbf{z}^{*}$ of system (22) is asymptotically stable, regardless of the fractional order $q \in(0,1)$.

Proof. We have:

$$
\begin{aligned}
\Re\left(\lambda_{p}\right) & =\Re(\alpha)+\Re\left(\beta \omega^{p}+\gamma \bar{\omega}^{p}\right)= \\
& =\Re(\alpha)+\Re\left((\beta+\bar{\gamma}) \omega^{p}\right) \leq \\
& =\Re(\alpha)+\left|(\beta+\bar{\gamma}) \omega^{p}\right|= \\
& =\Re(\alpha)+|\beta+\bar{\gamma}|<0,
\end{aligned}
$$

for any $p \in\{0,1, . ., n-1\}$, and hence, all eigenvalues of the Jacobian matrix $J\left(\mathbf{z}^{*}\right)$ are in the left half-plane. We obtain that $\mathbf{z}^{*}$ is asymptotically stable, regardless of the fractional order $q \in(0,1)$.

Moreover, based on Proposition 1, the following result holds: 
Proposition 4. If conditions 24), (25) and (26) are satisfied and

$$
\Re(\alpha)+|\beta+\bar{\gamma}| \geq 0,
$$

the steady state $\mathbf{z}^{*}$ of system (22) is asymptotically stable if and only if

$$
q<\frac{2}{\pi} \min _{p=\overline{0, n-1}}\left|\arg \left(\alpha+\beta \omega^{p}+\gamma \bar{\omega}^{p}\right)\right|
$$

where $\omega=\exp \left(\frac{2 \pi i}{n}\right)$. If the critical value

$$
q^{\star}=\frac{2}{\pi} \min _{p=\overline{0, n-1}}\left|\arg \left(\alpha+\beta \omega^{p}+\gamma \bar{\omega}^{p}\right)\right| \in(0,1)
$$

a Hopf bifurcation is expected to occur in system (22) in a neighborhood of the steady state $\mathbf{z}^{*}$.

Taking into consideration the fact that the parameters $\alpha, \beta$ and $\gamma$ given by (24), (25) and (26) belong to the complex plane, due to the high complexity of the problem, it is a very difficult task to determine a simple expression for the value of the minimum involved in the inequality (30) or the formula of the critical value $q^{\star}$. In the particular case when $\alpha, \beta$ and $\gamma$ take real values, we refer to Kaslik and Sivasundaram (2012b) for a more detailed analysis.

In the following, we exemplify a particular complex-valued case which will be investigated in detail.

Example 2. For simplicity, let us consider $\mathbf{z}^{*}=\mathbf{0}$. Moreover, we assume that the self-regulating parameters of the neurons are equal to 1 , no neuronal self-connection is present, and $\beta$ and $\gamma$ belong to the unit circle, i.e.:

$$
\alpha=-1, \quad \beta=\exp \left(i \theta_{1}\right), \quad \gamma=\exp \left(i \theta_{2}\right), \quad \text { where } \theta_{1}, \theta_{2} \in(-\pi, \pi] .
$$

Let $S_{q}\left(\mathbf{z}^{*}\right) \subset(-\pi, \pi] \times(-\pi, \pi]$ denote the set of parameters $\left(\theta_{1}, \theta_{2}\right)$ for which the equilibrium state $\mathbf{z}^{*}=\mathbf{0}$ is an asymptotically stable equilibrium state of system (22). The set $S_{q}\left(\mathbf{z}^{*}\right)$ is called stability domain of the equilibrium state $\mathbf{z}^{*}$ of system (22), with respect to the fractional order $q$.

It can be easily seen that the eigenvalues $\lambda_{p}, p=\overline{0, n-1}$, of the Jacobian matrix $J\left(\mathbf{z}^{*}\right)$ can be expressed as:

$$
\lambda_{p}=-1+2 \cos \left(\frac{\theta_{1}-\theta_{2}}{2}+\frac{2 p \pi}{n}\right) \exp \left(i \frac{\theta_{1}+\theta_{2}}{2}\right)
$$



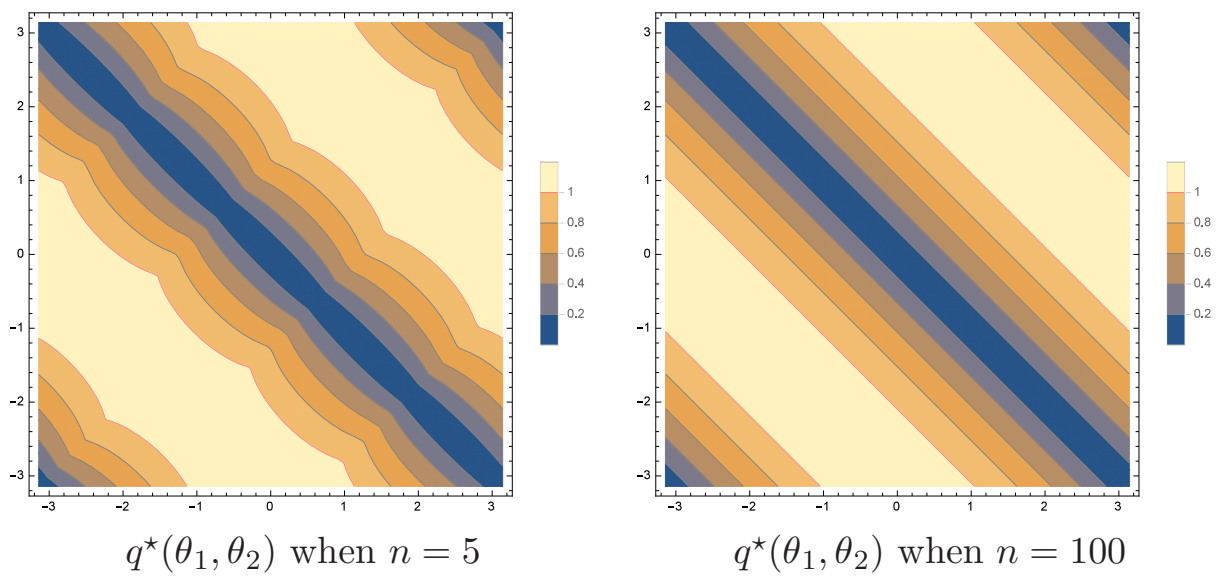

Figure 2: Density plot of critical values $q^{\star}$ with respect to $\left(\theta_{1}, \theta_{2}\right)$, when $\alpha=-1, \beta=$ $\exp \left(i \theta_{1}\right), \gamma=\exp \left(i \theta_{2}\right)$, for a small number of neurons $(n=5$, left) and for a large number of neurons ( $n=100$, right), respectively.

Based on Proposition 3, we deduce that if $\cos \left(\theta_{1}+\theta_{2}\right)<-\frac{1}{2}$ (or equivalently $\left.\frac{2 \pi}{3}<\left|\theta_{1}+\theta_{2}\right|<\frac{4 \pi}{3}\right)$ then $\Re\left(\lambda_{p}\right)<0$ and therefore, the steady state $\mathbf{z}^{*}$ is asymptotically stable, for any $q \in(0,1]$. Therefore, we deduce:

$D_{\theta_{1}, \theta_{2}}=\left\{\left(\theta_{1}, \theta_{2}\right) \in(-\pi, \pi] \times(-\pi, \pi]: \frac{2 \pi}{3}<\left|\theta_{1}+\theta_{2}\right|<\frac{4 \pi}{3}\right\} \subset S_{1}\left(\mathbf{z}^{*}\right) \subset S_{q}\left(\mathbf{z}^{*}\right), \quad \forall q \in(0,1]$.

Fig. 2 shows that as the number of neurons $n$ from the ring increases, the stability domain $S_{1}\left(\mathbf{z}^{*}\right)$ approaches the set $D_{\theta_{1}, \theta_{2}}$ (light tan colored region). In the dark blue region we have $q^{\star}<0.2$, meaning that the steady state $\mathbf{z}^{*}$ is asymptotically stable only for small values of the fractional order $q$. It can be observed that as the combination of parameters $\left(\theta_{1}, \theta_{2}\right)$ approaches the line $\theta_{1}+\theta_{2}=0$, the critical value $q^{\star}$ decreases towards 0 . In fact, if $\theta_{1}+\theta_{2}=0$ and the number of neurons $n$ is sufficiently large, the steady state $\mathbf{z}^{*}$ is unstable for any $q \in(0,1]$. Indeed, as

$$
\lambda_{p}=-1+2 \cos \left(\theta_{1}+\frac{2 p \pi}{n}\right) \in \mathbb{R}, \quad \forall p=\overline{1, n}
$$

when $n$ is sufficiently large, it can be easily shown that there exists $p \in$ $\{0,1, \ldots, n-1\}$ such that $\lambda_{p}>0$.

In the following, we exemplify a complex-valued neural network with an infinity of steady states. 
Example 3. We consider the following complex-valued neural network of $n=3$ neurons with ring structure:

$$
\left\{\begin{array}{l}
{ }^{c} D^{q} z_{1}(t)=-z_{1}(t)+T_{0} g\left(z_{1}(t)\right)+T_{1} g\left(z_{2}(t)\right)+T_{2} g\left(z_{3}(t)\right) \\
{ }^{c} D^{q} z_{2}(t)=-z_{2}(t)+T_{2} g\left(z_{1}(t)\right)+T_{0} g\left(z_{2}(t)\right)+T_{1} g\left(z_{3}(t)\right) \\
{ }^{c} D^{q} z_{3}(t)=-z_{3}(t)+T_{1} g\left(z_{1}(t)\right)+T_{2} g\left(z_{2}(t)\right)+T_{0} g\left(z_{3}(t)\right)
\end{array}\right.
$$

In this neural network, $a=1, T_{11}=T_{22}=T_{33}=T_{0}=1-2 i, T_{12}=T_{23}=$ $T_{31}=T_{1}=1+i$ and $T_{13}=T_{21}=T_{32}=T_{2}=i$. The activation function $i s$ $g(z)=\frac{z}{1+|z|}$.

The state $\left(z_{1}, z_{2}, z_{3}\right)^{T}$ is a steady state of system (31) if and only if it is a solution of the following nonlinear algebraic system with complex coefficients:

$$
\left\{\begin{array}{l}
z_{1}=T_{0} g\left(z_{1}\right)+T_{1} g\left(z_{2}\right)+T_{2} g\left(z_{3}\right) \\
z_{2}=T_{2} g\left(z_{1}\right)+T_{0} g\left(z_{2}\right)+T_{1} g\left(z_{3}\right) \\
z_{3}=T_{1} g\left(z_{1}\right)+T_{2} g\left(z_{2}\right)+T_{0} g\left(z_{3}\right)
\end{array}\right.
$$

From the first equation of (32) and $|g(z)| \leq 1$, it follows that

$$
\left|z_{1}\right| \leq\left|T_{0}\right|+\left|T_{1}\right|+\left|T_{2}\right|=1+\sqrt{2}+\sqrt{5}=r .
$$

We get similar results for $\left|z_{2}\right|$ and $\left|z_{3}\right|$, and hence, all the steady states of system (31) are inside the bounded set $B(0, r) \times B(0, r) \times B(0, r) \subset \mathbb{C}^{3}$, where $B(0, r)$ denotes the ball centered at the origin, of radius $r=1+\sqrt{2}+\sqrt{5}$ from the complex plane.

Particular steady states of system (31) with equal components, i.e. of the form $\left(z^{*}, z^{*}, z^{*}\right)^{T}$, can be found by solving the equation

$$
-z+\left(T_{0}+T_{1}+T_{2}\right) g(z)=0
$$

which is equivalent to $z=2 g(z)$. It follows that $\left(z^{*}, z^{*}, z^{*}\right)^{T}$ is a steady state of system (31) if and only if either $z^{*}=0$ (trivial equilibrium) or $\left|z^{*}\right|=1$ (i.e $z^{*}$ belongs to the unit circle of the complex plane). We deduce that there are infinitely many steady states for the system (31).

In particular, we focus our attention on the stability of the trivial steady state $\mathbf{z}^{*}=(0,0,0)^{T}$. As $g^{\prime}(0)=1$, based on (24), (25) and (26), we obtain $\alpha=-2 i, \beta=T_{1}=1+i$ and $\gamma=T_{2}=i$. The first eigenvalue of the Jacobian matrix is $\lambda_{0}=\alpha+\beta+\gamma=1$, and therefore, the trivial solution is unstable for any fractional order $q \in(0,1)$. Indeed, numerical computations (see Fig. 3) show that the trajectory of the system with an initial condition from a small neighborhood of the trivial steady state converges to one of the steady states of the form $\left(z^{*}, z^{*}, z^{*}\right)^{T}$ with $\left|z^{*}\right|=1$. 


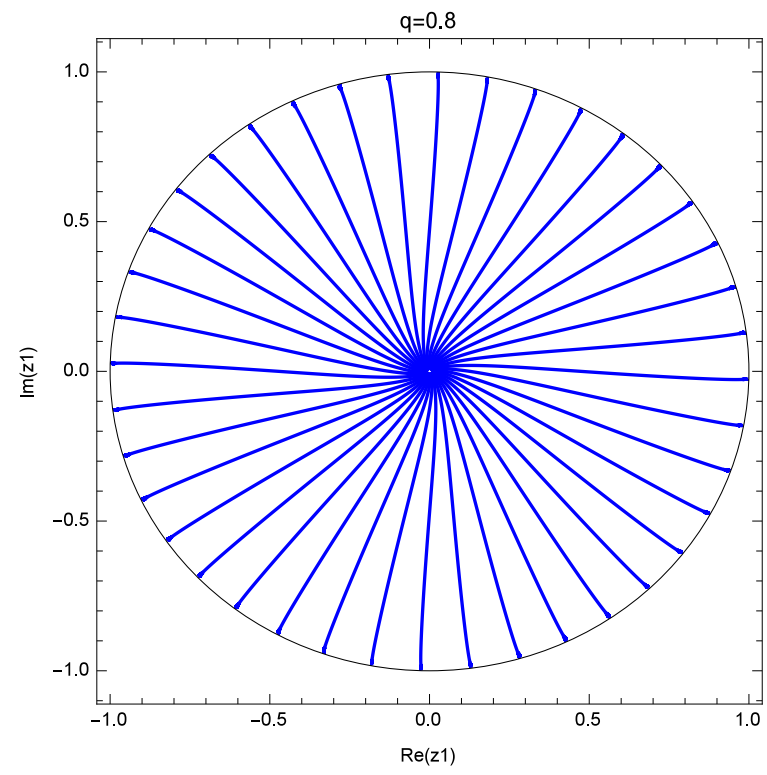

Figure 3: Trajectories of system (31) with fractional order $q=0.8$ and initial conditions from a small neighborhood of the null solution.

\section{Conclusions}

In the case of fractional-order complex-valued neural networks with ring or hub structure, conditions for the stability and instability of a steady state have been explored and various values of the fractional order $q \in(0,1)$ for which Hopf bifurcations may occur, have been identified. Theoretical and numerical results presented in this paper show that fractional-order complexvalued neural networks may exhibit rich dynamical behavior in a neighborhood of a steady state, ranging from stability, quasi-periodicity occurring due to a supercritical Hopf bifurcation, as well as instability. Moreover, it has been pointed out that complex-valued neural networks may possess an infinite number of steady states.

It is worth noting that chaotic behaviour has not been observed in our numerical simulations. This may be partly due to the activation function which has been used in the examples, as well as to the fact that in complex valued systems, a large number of steady states may be present (in our last example, we have an infinity of steady states). At least a part of these steady states may be asymptotically stable, such that the union of their domains of attraction include the whole phase-space. Assessment of chaotic behavior in fractional- or integer-order complex-valued neural networks with or without 
delays will be the topic of a future paper.

These simple neural network structures can be regarded as a test bed for understanding the dynamics of more complicated structures. Applications of such neural networks in pattern recognition and classification, intelligent image processing, nonlinear filtering, brain-computer interfaces or time series prediction may also constitute challenging directions for future research.

The extension of the results presented in this paper to more general fractional-order quaternion-valued neural networks Liu et al. (2016b), Cliffordvalued neural networks Liu et al. (2016a) or complex-valued switched interval neural networks Cao et al. (2013) is also an area worth exploring in future works.

\section{Acknowledgement}

The authors are grateful to the editor and the referees for their helpful comments and suggestions which have improved the quality of the paper.

This work was supported by a grant of the Romanian National Authority for Scientific Research and Innovation, CNCS-UEFISCDI, project no. PNII-RU-TE-2014-4-0270.

\section{Appendix A. Proof of Lemma 1.}

We first assume that $A=\theta_{1}-\frac{\theta_{2}}{2} \notin\left\{-\pi,-\frac{\pi}{2}, 0, \frac{\pi}{2}, \pi\right\}$.

Let us denote the roots of the polynomial $P(\lambda)$ by $\lambda_{1}=r_{1} e^{i \phi_{1}}$ and $\lambda_{2}=r_{2} e^{i \phi_{2}}$, with $r_{1}, r_{2}>0$ and $\phi_{1}, \phi_{2} \in(-\pi, \pi]$. We may assume, without loss of generality, that $\phi_{1} \geq \phi_{2}$. In this case, Vieta's formulas are:

$$
\left\{\begin{array}{l}
\lambda_{1}+\lambda_{2}=\alpha+\beta=\rho_{1} e^{i \theta_{1}} \\
\lambda_{1} \cdot \lambda_{2}=(\alpha \beta-\gamma)=\rho_{2} e^{i \theta_{2}}
\end{array}\right.
$$

Taking the absolute value in the second formula, it follows that $r_{1} r_{2}=\rho_{2}$ and hence, the second Vieta's formula simplifies to

$$
e^{i\left(\phi_{1}+\phi_{2}\right)}=e^{i \theta_{2}}
$$

which leads to $\phi_{1}+\phi_{2}=\theta_{2}+2 k \pi$, where $k \in \mathbb{Z}$. However, since $\phi_{1}+\phi_{2} \in$ $(-2 \pi, 2 \pi]$ and $\theta_{2} \in(-\pi, \pi]$, it follows that only three distinct cases have to be considered: $k=-1$ (if $\theta_{2}>0$ ), $k=0$ and $k=1$ (if $\theta_{2}<0$ ).

Denoting $\phi=\phi_{1}-\phi_{2} \in[0,2 \pi)$, and dividing the first Vieta's formula by $(-1)^{k} e^{i \theta_{2} / 2}$, we obtain:

$$
r_{1} e^{i \phi / 2}+r_{2} e^{-i \phi / 2}=(-1)^{k} \rho_{1} e^{i A} .
$$


Taking the real part, it follows that

$$
\left(r_{1}+r_{2}\right) \cos (\phi / 2)=(-1)^{k} \rho_{1} \cos (A)
$$

and therefore, $\cos (\phi / 2)$ and $(-1)^{k} \cos (A)$ must have the same sign, and hence, as $\phi \in[0,2 \pi)$ and $\operatorname{sign}(\sin (\phi))=\operatorname{sign}(\cos (\phi / 2))$, it follows that:

$$
\operatorname{sign}\left((-1)^{k} \sin (\phi)\right)=\operatorname{sign}(\cos (A)) .
$$

Multiplying eq. (A.1) by $e^{i \phi / 2}$ and taking the imaginary part of the resulting equation, we obtain:

$$
r_{1} \sin (\phi)=(-1)^{k} \rho_{1} \sin \left(A+\frac{\phi}{2}\right)
$$

Similarly, multiplying eq. (A.1) by $e^{-i \phi / 2}$ and taking the imaginary part of the resulting equation, we obtain:

$$
-r_{2} \sin (\phi)=(-1)^{k} \rho_{1} \sin \left(A-\frac{\phi}{2}\right)
$$

As $r_{1}, r_{2}, \rho_{1}>0$, it follows that the following condition must be satisfied:

$$
\operatorname{sign}\left((-1)^{k} \sin (\phi)\right)=\operatorname{sign}\left(\sin \left(\frac{\phi}{2} \pm A\right)\right) .
$$

Combining the last two equalities, we get:

$$
-r_{1} r_{2} \sin ^{2}(\phi)=\rho_{1}^{2} \sin \left(A+\frac{\phi}{2}\right) \sin \left(A-\frac{\phi}{2}\right)
$$

or equivalently, since $r_{1} r_{2}=\rho_{2}$,

$$
2 \rho_{2}\left(\cos ^{2}(\phi)-1\right)=\rho_{1}^{2}(\cos (\phi)-\cos (2 A)) .
$$

Therefore, $\cos (\phi)$ is a root of the polynomial

$$
p(x)=x^{2}-u x+u \cos (2 A)-1, \quad \text { where } u=\frac{\rho_{1}^{2}}{2 \rho_{2}} .
$$

The discriminant of the second degree polynomial $p(x)$ is

$$
\Delta=u^{2}-4 u \cos (2 A)+4 \geq(u-2)^{2} \geq 0 .
$$


We have

$$
\begin{aligned}
p(-1) & =u[1+\cos (2 A)]>0 \\
p(\cos (2 A)) & =\cos ^{2}(2 A)-1<0 \\
p(1) & =u(\cos (2 A)-1)<0
\end{aligned}
$$

and hence, the smallest root of the polynomial $q(x)$ belongs to the interval $(-1, \cos (2 A))$, while the largest root is greater than 1 . Therefore, we obtain

$$
\cos (\phi)=\frac{u-\sqrt{u^{2}-4 u \cos (2 A)+4}}{2} \in(-1, \cos (2 A)) .
$$

and hence,

$$
\phi= \pm \arccos \left(\frac{u-\sqrt{u^{2}-4 u \cos (2 A)+4}}{2}\right)+2 l \pi= \pm 2 B+2 l \pi, \quad l \in \mathbb{Z} .
$$

As $\cos (\phi)<\cos (2 A)$, we get $\cos (2 B)<\cos (2 A)$, and therefore,

$$
\sin (A+B) \sin (B-A)>0,
$$

which means that $\sin (B \pm A)$ have the same signs. Moreover, as $B \in\left(0, \frac{\pi}{2}\right)$, it follows that

$$
\operatorname{sign}(\sin (B \pm A))=\operatorname{sign}(\sin B \cos A \pm \cos B \sin A)=\operatorname{sign}(\cos A) .
$$

Moreover, since $\phi \in[0,2 \pi)$, it follows that we may have two cases:

Case 1. $\phi=2 B \in[0, \pi)$. In this case, it is easy to see that (A.2) and (A.4) imply (A.3). Moreover:

- If $k=-1$ (only if $\theta_{2}>0$ ), we obtain $\phi_{1,2}=\frac{\theta_{2}}{2}-\pi \pm B$. It is easy to see that $\phi_{1,2} \in(-\pi, \pi]$ if and only if $\theta_{2}>2 B$. Based on (A.2), this subcase holds only if $\cos (A)<0$, leading to (b.3).

- If $k=0$, we obtain $\phi_{1,2}=\frac{\theta_{2}}{2} \pm B \in(-\pi, \pi]$. Based on (A.2), this subcase holds only if $\cos (A)>0$. Therefore, we obtain case (a).

- If $k=1$ (only if $\theta_{2}<0$ ), we obtain $\phi_{1,2}=\frac{\theta_{2}}{2}+\pi \pm B$. It is easy to see that $\phi_{1,2} \in(-\pi, \pi]$ if and only if $\theta_{2} \leq-2 B$. Based on (A.2), this subcase holds only if $\cos (A)<0$, leading to (b.1). 
Case 2. $\phi=2 \pi-2 B \in[\pi, 2 \pi)$. In this case, based on (A.2) and (A.4), we obtain:

$$
\begin{aligned}
\operatorname{sign}\left(\sin \left(\frac{\phi}{2} \pm A\right)\right) & =\operatorname{sign}(\sin (\pi-B \pm A)) \\
& =\operatorname{sign}(\sin (B \pm A))=\operatorname{sign}(\cos (A)) \\
& =\operatorname{sign}\left((-1)^{k} \sin (\phi)\right)
\end{aligned}
$$

and hence, condition (A.3) is satisfied. Moreover,

- If $k=-1$ (only if $\theta_{2}>0$ ), we obtain $\phi_{1,2}=\frac{\theta_{2}}{2}-\pi \pm(\pi-B)$. We observe that $\phi_{2}=\frac{\theta_{2}}{2}+B-2 \pi<-\pi$, which is absurd.

- If $k=0$, we obtain $\phi_{1,2}=\frac{\theta_{2}}{2} \pm(\pi-B)$. It is easy to see that $\phi_{1,2} \in(-\pi, \pi]$ if and only if $-2 B<\theta_{2} \leq 2 B$. Based on (A.2), this subcase holds only if $\cos (A)<0$, leading to (b.2).

- If $k=1$ (only if $\theta_{2}<0$ ), we obtain $\phi_{1,2}=\frac{\theta_{2}}{2}+\pi \pm(\pi-B)$. We observe that $\phi_{1}=\frac{\theta_{2}}{2}-B+2 \pi>\pi$, which is absurd.

If $A=\theta_{1}-\frac{\theta_{2}}{2} \in\left\{-\frac{\pi}{2}, \frac{\pi}{2}\right\}$, i.e. $\cos (A)=0$, it follows from (A.1) that

$$
\left(r_{1}+r_{2}\right) \cos (\phi / 2)=0
$$

and hence, we obtain $\phi_{1}-\phi_{2}=\phi=\pi$. Taking into account that $\phi_{1}+\phi_{2}=$ $\theta_{2}+2 k \pi$, the only possible solution is $\phi_{1,2}=\frac{\theta_{2} \pm \pi}{2}$. This is a special case included in point (a) of the Lemma.

If $A=\theta_{1}-\frac{\theta_{2}}{2} \in\{-\pi, 0, \pi\}$, i.e. $\cos (A)=(-1)^{p}, \sin (A)=0$, taking the real and imaginary parts of (A.1) we obtain

$$
\left\{\begin{array}{l}
\left(r_{1}+r_{2}\right) \cos (\phi / 2)=(-1)^{k+p} \rho_{1} \\
\left(r_{1}-r_{2}\right) \sin (\phi / 2)=0
\end{array}\right.
$$

From here, either $r_{1}=r_{2}$ or $\phi=0$.

In the first case, $r_{1}=r_{2}$, taking into account that $r_{1} r_{2}=\rho_{2}$, we obtain $r_{1}=r_{2}=\sqrt{\rho_{2}}$ and

$$
\cos (\phi / 2)=(-1)^{k+p} \frac{\rho_{1}}{2 \sqrt{\rho_{2}}} .
$$

and hence,

$$
\cos (\phi)=2 \cos ^{2}(\phi / 2)-1=\frac{\rho_{1}^{2}}{2 \rho_{2}}-1=u-1 .
$$


We observe that this can only hold if and only if $\rho_{1}^{2} \leq 4 \rho_{2}$. Moreover, as $\phi \in[0,2 \pi)$, we have $\phi=\arccos (u-1)=2 B$ or $\phi=2 \pi-\arccos (u-1)=$ $2(\pi-B)$. From here, the proof follows cases 1 and 2 as above.

In the second case, $\phi=0$, the above system shows that $k+p$ is an even number.

If $A=0$, then $k=0$ and it simply follows $\phi_{1,2}=\frac{\theta_{2}}{2}$, which is a particular case of point (a) of the Lemma.

If $A \in\{-\pi, \pi\}$, then $k= \pm 1$ and hence $\phi_{1,2}=\frac{\theta_{2}}{2}+\pi$ if $\theta_{2}<0$ and

$\phi_{1,2}=\frac{\theta_{2}}{2}-\pi$ if $\theta_{2}>0$, in agreement with points (b.1) and (b.3) of the Lemma.

\section{References}

NanoDotTek, TM, 2007. What is fractance and why is it useful? Tech. Rep. NDT24-11-2007, p.//www. nanodottek.com.

Anastasio, T., 1994. The fractional-order dynamics of brainstem vestibulooculomotor neurons. Biological Cybernetics 72 (1), 69-79.

Arena, P., Fortuna, L., Porto, D., 2000. Chaotic behavior in noninteger-order cellular neural networks. Physical Review E 61 (1), 776-781.

Baldi, P., Atiya, A. F., 1994. How delays affect neural dynamics and learning. IEEE Transactions on Neural Networks 5 (4), 612-621.

Bao, H., Park, J. H., Cao, J., 2016. Synchronization of fractional-order complex-valued neural networks with time delay. Neural Networks 81, $16-28$.

Barabási, A.-L., Albert, R., 1999. Emergence of scaling in random networks. Science 286 (5439), 509-512.

Boroomand, A., Menhaj, M., 2009. Fractional-order Hopfield neural networks. Lecture Notes in Computer Science 5506 LNCS (PART 1), 883890 .

Bungay, S. D., Campbell, S. A., 2007. Patterns of oscillation in a ring of identical cells with delayed coupling. International Journal of Bifurcation and Chaos 17 (9), 3109-3125.

Campbell, S. A., Ruan, S., Wei, J., 1999. Qualitative analysis of a neural network model with multiple time delays. International Journal of Bifurcation and Chaos in Applied Sciences and Engineering 9 (8), 1585-1595. 
Cao, J., Alofi, A., Al-Mazrooei, A., Elaiw, A., 2013. Synchronization of switched interval networks and applications to chaotic neural networks. In: Abstract and Applied Analysis. Vol. 2013. Hindawi Publishing Corporation.

Chen, J., Zeng, Z., Jiang, P., 2014. Global Mittag-Leffler stability and synchronization of memristor-based fractional-order neural networks. Neural Networks 51, 1-8.

Chen, L., Chai, Y., Wu, R., Ma, T., Zhai, H., 2013. Dynamic analysis of a class of fractional-order neural networks with delay. Neurocomputing 111, 190-194.

Cottone, G., Paola, M. D., Santoro, R., 2010. A novel exact representation of stationary colored gaussian processes (fractional differential approach). Journal of Physics A: Mathematical and Theoretical 43 (8), 085002.

URL http://stacks . iop.org/1751-8121/43/i=8/a=085002

Diethelm, K., Ford, N., Freed, A., 2002. A predictor-corrector approach for the numerical solution of fractional differential equations. Nonlinear Dynamics 29 (1-4), 3-22.

El-Saka, H., Ahmed, E., Shehata, M., El-Sayed, A., 2009. On stability, persistence, and Hopf bifurcation in fractional order dynamical systems. Nonlinear Dynamics 56 (1-2), 121-126.

Elwakil, A., 2010. Fractional-order circuits and systems: An emerging interdisciplinary research area. IEEE Circuits and Systems Magazine 10 (4), $40-50$.

Engheia, N., 1997. On the role of fractional calculus in electromagnetic theory. IEEE Antennas and Propagation Magazine 39 (4), 35-46.

Georgiou, G. M., Koutsougeras, C., 1992. Complex domain backpropagation. IEEE transactions on Circuits and systems II: analog and digital signal processing 39 (5), 330-334.

Gray, R. M., 2005. Toeplitz and Circulant Matrices: A Review. Now Publishers Inc.

Guo, S., 2005. Spatio-temporal patterns of nonlinear oscillations in an excitatory ring network with delay. Nonlinearity 18 (5), 2391-2407. 
Guo, S., Huang, L., 2003. Hopf bifurcating periodic orbits in a ring of neurons with delays. Physica D: Nonlinear Phenomena 183 (1-2), 19-44.

Guo, S., Huang, L., 2006. Non-linear waves in a ring of neurons. IMA Journal of Applied Mathematics (Institute of Mathematics and Its Applications) 71 (4), 496-518.

Guo, S., Huang, L., 2007a. Stability of nonlinear waves in a ring of neurons with delays. Journal of Differential Equations 236 (2), 343-374.

Guo, S. J., Huang, L. H., 2007b. Pattern formation and continuation in a trineuron ring with delays. Acta Mathematica Sinica, English Series 23 (5), 799-818.

Henry, B., Wearne, S., 2002. Existence of Turing instabilities in a two-species fractional reaction-diffusion system. SIAM Journal on Applied Mathematics $62,870-887$.

Heymans, N., Bauwens, J.-C., 1994. Fractal rheological models and fractional differential equations for viscoelastic behavior. Rheologica Acta 33, 210-219.

Hirose, A., 2006. Complex-valued neural networks. Springer Science \& Business Media.

Hirose, A., 2009. Complex-valued neural networks: the merits and their origins. In: 2009 International Joint Conference on Neural Networks. IEEE, pp. 1237-1244.

Hirsch, M., 1989. Convergent activation dynamics in continuous-time networks. Neural Networks 2, 331-349.

Huang, C., Cao, J., Xiao, M., Alsaedi, A., Hayat, T., 2017. Bifurcations in a delayed fractional complex-valued neural network. Applied Mathematics and Computation 292, 210-227.

Ichise, M., Nagayanagi, Y., Kojima, T., 1971. An analog simulation of noninteger order transfer functions for analysis of electrode processes. Journal of Electroanalytical Chemistry 33, 253-265.

Kaslik, E., 2009. Dynamics of a discrete-time bidirectional ring of neurons with delay. In: Proceedings of the International Joint Conference on Neural Networks, Atlanta, GA, USA. pp. 1539-1546. 
Kaslik, E., Balint, S., 2009. Complex and chaotic dynamics in a discretetime-delayed Hopfield neural network with ring architecture. Neural Networks 22 (10), 1411-1418.

Kaslik, E., Sivasundaram, S., 2011. Dynamics of fractional-order neural networks. In: Proceedings of the International Joint Conference on Neural Networks, San Jose, California, USA, July 31-August 5, 2011. IEEE Computer Society Press, pp. 611-618.

Kaslik, E., Sivasundaram, S., 2012a. Non-existence of periodic solutions in fractional-order dynamical systems and a remarkable difference between integer and fractional-order derivatives of periodic functions. Nonlinear Analysis: Real World Applications 13 (3), 1489-1497.

Kaslik, E., Sivasundaram, S., 2012b. Nonlinear dynamics and chaos in fractional-order neural networks. Neural Networks 32, 245-256.

Kaslik, E., Sivasundaram, S., 2014. Differences between fractional-and integer-order dynamics. In: 10th International Conference on Mathematical Problems in Engineering, Aerospace and Sciences: ICNPAA 2014. Vol. 1637. AIP Publishing, pp. 479-486.

Kilbas, A., Srivastava, H., Trujillo, J., 2006. Theory and Applications of Fractional Differential Equations. Elsevier.

Kitajima, H., Kurths, J., 2009. Bifurcation in neuronal networks with hub structure. Physica A: Statistical Mechanics and its Applications 388 (20), 4499-4508.

Kra, I., Simanca, S. R., 2012. On circulant matrices. Notices of the AMS 59 (3), 368-377.

Kuroe, Y., Yoshid, M., Mori, T., 2003. On activation functions for complexvalued neural networks existence of energy functions. In: Artificial Neural Networks and Neural Information ProcessingICANN/ICONIP 2003. Springer, pp. 985-992.

Lakshmikantham, V., Leela, S., Devi, J. V., 2009. Theory of fractional dynamic systems. Cambridge Scientific Publishers.

Li, C., Ma, Y., 2013. Fractional dynamical system and its linearization theorem. Nonlinear Dynamics 71 (4), 621-633. 
Liu, Y., Xu, P., Lu, J., Liang, J., 2016a. Global stability of Clifford-valued recurrent neural networks with time delays. Nonlinear Dynamics 84 (2), $767-777$.

Liu, Y., Zhang, D., Lu, J., Cao, J., 2016b. Global $\mu$-stability criteria for quaternion-valued neural networks with unbounded time-varying delays. Information Sciences 360, 273-288.

Lu, X., Guo, S., 2008. Complete classification and stability of equilibria in a delayed ring network. Electronic Journal of Differential Equations 2008, $1-12$.

Lundstrom, B., Higgs, M., Spain, W., Fairhall, A., 2008. Fractional differentiation by neocortical pyramidal neurons. Nature Neuroscience 11 (11), $1335-1342$.

Mainardi, F., 1996. Fractional relaxation-oscillation and fractional phenomena. Chaos Solitons Fractals 7 (9), 1461-1477.

Matignon, D., 1996. Stability results for fractional differential equations with applications to control processing. In: Computational Engineering in Systems Applications. pp. 963-968.

Matsuzaki, T., Nakagawa, M., 2003. A chaos neuron model with fractional differential equation. Journal of the Physical Society of Japan 72 (10), $2678-2684$.

Metzler, R., Klafter, J., 2000. The random walk's guide to anomalous diffusion: a fractional dynamics approach. Physics Reports 339 (1), 1 - 77.

Milo, R., Shen-Orr, S., Itzkovitz, S., Kashtan, N., Chklovskii, D., Alon, U., 2002. Network motifs: simple building blocks of complex networks. Science 298.

Nakagawa, M., Sorimachi, K., 1992. Basic characteristics of a fractance device. IEICE Transactions on Fundamentals of Electronics, Communications and Computer Sciences E75-A (12), 1814-1819.

Petras, I., 2006. A note on the fractional-order cellular neural networks. In: IEEE International Conference on Neural Networks. pp. 1021-1024.

Podlubny, I., 1999. Fractional differential equations. Academic Press. 
Rakkiyappan, R., Cao, J., Velmurugan, G., 2015a. Existence and uniform stability analysis of fractional-order complex-valued neural networks with time delays. Neural Networks and Learning Systems, IEEE Transactions on $26(1), 84-97$.

Rakkiyappan, R., Sivaranjani, R., Velmurugan, G., Cao, J., 2016. Analysis of global o (t- $\alpha$ ) stability and global asymptotical periodicity for a class of fractional-order complex-valued neural networks with time varying delays. Neural Networks.

Rakkiyappan, R., Velmurugan, G., Cao, J., 2014. Finite-time stability analysis of fractional-order complex-valued memristor-based neural networks with time delays. Nonlinear Dynamics 78 (4), 2823-2836.

Rakkiyappan, R., Velmurugan, G., Cao, J., 2015b. Stability analysis of fractional-order complex-valued neural networks with time delays. Chaos, Solitons \& Fractals 78, 297-316.

Sabatier, J., Farges, C., 2012. On stability of commensurate fractional order systems. International Journal of Bifurcation and Chaos 22 (04), 1250084.

Sugimoto, N., 1991. Burgers equation with a fractional derivative; hereditary effects on nonlinear acoustic waves. Journal of Fluid Mechanics 225, 631653 .

Wang, H., Yu, Y., Wen, G., 2014a. Stability analysis of fractional-order Hopfield neural networks with time delays. Neural Networks 55, 98-109.

Wang, H., Yu, Y., Wen, G., 2014b. Stability analysis of fractional-order Hopfield neural networks with time delays. Neural Networks 55, 98-109.

Wang, H., Yu, Y., Wen, G., Zhang, S., 2015. Stability analysis of fractionalorder neural networks with time delay. Neural Processing Letters 42 (2), 479-500.

Wei, J., Jiang, W., 2006. Stability and bifurcation analysis in a neural network model with delays. Dynamics of Continuous, Discrete and Impulsive Systems Series A: Mathematical Analysis 13 (2), 177-192.

Xiao, M., Zheng, W. X., Jiang, G., Cao, J., 2015. Undamped oscillations generated by Hopf bifurcations in fractional-order recurrent neural networks with Caputo derivative. Neural Networks and Learning Systems, IEEE Transactions on 26 (12), 3201-3214. 
Zhou, S., Hu, P., Li, H., 2009. Chaotic synchronization of a fractional neuron network system with time-varying delays. In: 2009 International Conference on Communications, Circuits and Systems, ICCCAS 2009. pp. 863867.

Zhou, S., Li, H., Zhu, Z., 2008. Chaos control and synchronization in a fractional neuron network system. Chaos, Solitons and Fractals 36 (4), 973-984.

Zhu, H., Zhou, S., Zhang, W., 2008. Chaos and synchronization of timedelayed fractional neuron network system. In: Proceedings of the 9th International Conference for Young Computer Scientists, ICYCS 2008. pp. 2937-2941. 